\title{
Multifaceted Hemolytic Uremic Syndrome in Pediatrics
}

\author{
Carla M. Nester \\ Departments of Internal Medicine and Pediatrics, Rare Renal Disease Clinic, University of lowa, lowa City, lowa, USA
}

\section{Key Words}

Hemolytic uremic syndrome - STEC HUS - Atypical

hemolytic uremic syndrome $\cdot$ Complement

\begin{abstract}
Hemolytic uremic syndromes can have devastating consequences in childhood. The common feature of a microangiopathic hemolytic anemia and thrombocytopenia associated with varying degrees of renal injury often creates diagnostic confusion. The inability to arrive at a definitive diagnosis quickly can lead to a delay in initiating renal-preserving and sometimes life-saving treatment. Currently, both the treatment plan and the prognosis vary substantially according to the presumed diagnosis. The availability of anti-complement therapy makes differentiating the cause of the hemolytic uremic syndrome particularly critical. Therefore, it is imperative that consideration be given to each of the possible syndromes at presentation in order to facilitate correct diagnosis and development of an appropriate treatment strategy for both the acute phase and for the long-term care of the patient.

Copyright $\odot 2013$ S. Karger AG, Basel
\end{abstract}

\section{Introduction}

The presentation of a microangiopathic hemolytic anemia, thrombocytopenia and renal impairment in childhood points to the diagnosis of one of the hemolytic uremic syndromes (HUSs). Collectively, the syndromes are characterized by a typical pathological lesion: endothelial cell swelling, subendothelial accumulation of cellular debris and platelet-rich thrombi. This pathological lesion leads to vascular lumen obstruction, provoking organ ischemia and the myriad of symptoms that are seen in HUS. The fact that each of these syndromes results from a near identical pathological lesion results in clinical overlap that can contribute to diagnostic confusion. Both the treatment plan and the prognosis vary substantially according to the presumed diagnosis. Therefore it is imperative that consideration be given to each of the possible syndromes at presentation in order to facilitate correct diagnosis and development of an appropriate treatment strategy in both the acute and long-term setting.

Historically, the HUSs in children have been classified as either diarrhea-positive versus diarrhea-negative or as typical versus atypical. These designations have now been supplanted by terms that are meant to be more descrip-

\section{KARGER}

Fax +4161306 1234

E-Mail karger@karger.com

www.karger.com
2013 S. Karger AG, Basel

0253-5068/13/0353-0086\$38.00/0

Accessible online at:

www.karger.com/bpu
Assist. Prof Carla Nester, MD, MSA

Pediatric Glomerular Disease Clinic

University of Iowa Hospitals and Clinics

200 Hawkins Drive, BT 4036, Iowa City, IA 52242-1081 (USA)

E-Mail carla-nester@uiowa.edu 
tive and include STEC HUS (for Shiga toxin-producing Escherichia coli) and complement-mediated HUS as well as multiple secondary forms that are referred to by their associated pathology (i.e. streptococcal-associated HUS, malignant hypertension-associated HUS, etc.) (table 1). Though the renal microvasculature is the predominant site of the pathological lesion in HUS, the vasculature of other organs are frequently involved and may play a significant role in the morbidity for any given patient. A brief overview of the syndromes is presented below.

\section{STEC HUS}

\section{Pathophysiology}

Shiga toxin (Stx) infection is a classic example of an infection that can induce a microangiopathic hemolytic anemia with thrombocytopenia. Bloody diarrhea is often the first clinical sign of disease and will not only be the clue to an inciting infection but will be the trigger for the necessary laboratories to facilitate a firm diagnosis. When acute kidney injury also occurs, the triad for the diagnosis of an HUS has been met and it becomes appropriate to define the patient as having STEC HUS.

The vast majority $(\sim 80 \%)$ of individuals with STEC infection experience a self-limited course of gastrointestinal symptoms and do not progress to the systemic symptoms of HUS $[1,2]$. The factors predisposing patients with STEC infection to the systemic syndrome of STEC HUS remains unclear.

Escherichia coli O157:H7 (also referred to as enterohemorrhagic E. coli - EHEC) is the prototypical pathogen known to cause STEC HUS in the United States, however Shigella and other E. coli have also been associated with disease in other settings [3-5]. The incidence of STEC HUS is estimated to be $1-2$ cases/ 100,000 per year, with most pediatric diagnoses occurring in patients under age 5 years $[6,7]$. The relative frequency of STEC HUS requires that it be the primary consideration when approaching the child with HUS.

The pathophysiology of STEC HUS relates to the biological characteristics of the causative organisms and the toxins they express. Following ingestion, non-invasive EHEC colonize the terminal ileum and releases Stx $[8,9]$. The ability to infect and cause death of enterocytes as well as trigger systemic intravascular effects is a direct consequence of the Stx. Stx binds to specific receptors distributed throughout endothelial cells of the gastrointestinal tract, pancreas, brain, and kidney and is responsible for a multitude of cytotoxic, proinflammatory and
Table 1. HUSs that can present in childhood - a comprehensive laboratory workup is designed to distinguish these entities from one another, particularly when the clinical presentation is not straightforward

STEC HUS (typical or diarrhea-positive HUS)

Toxin associated

Secondary HUS

Streptococcal infection associated

Malignant hypertension associated

Pancreatitis associated

Medication associated

Transplant related

Autoimmune disorder associated

Cobalamin disorder associated

Septicemia or DIC associated

Malignancy associated

Pregnancy associated (HELLP)

Complement-mediated HUS (atypical or diarrhea-negative HUS)

Alternate complement pathway gene abnormality Idiopathic

prothrombotic effects [10]. Systemic complications resulting from Stx include cell death, platelet activation and deposition, cytokine release and increased tissue factor expression on the cells of the microvasculature [11]. Microthrombi formation in the microvasculature following Stx-induced platelet activation results in the abnormal shear stress and disturbances in blood flow that eventually leads to the production of the fragmented red blood cells or schistocytes representative of a microangiopathic anemia.

\section{Clinical Manifestations and Diagnosis}

Children with STEC HUS classically present with bloody diarrhea and then are found to have a microangiopathic anemia (often associated with an elevated lactic acid dehydrogenase and a decreased haptoglobin with no signs of autoimmunity), thrombocytopenia and acute kidney injury as evidenced by elevation of serum creatinine above the upper limit for age. It is important to note that while more than $90 \%$ of children with STEC HUS will experience bloody diarrhea, a subset of patients will not [1]. The enteric prodrome often lasts 4-7 days prior to the development of STEC HUS. Stool culture positivity for Stx-producing E. coli aids in diagnosis, however a negative stool culture does not rule out STEC HUS in the presence of the above classical presentation. Thrombocytopenia is the first blood abnormality often present in patients and is subsequently followed by hemolysis and 
anemia precipitated by the small vessel thrombi [1]. Other symptoms of STEC HUS include oligoanuria, neurological dysfunction (ranging from transient ischemic attacks to seizures), pancreatitis, and elevated liver enzymes. Fever may be present in STEC HUS. Metabolic abnormalities are common and include hyperkalemia, hyponatremia, and hyperuricemia. Severe hemorrhagic colitis may lead to gangrenous colitis [12], bowel perforation, and sepsis in the most severe cases [13].

Acute kidney injury is a classic component of STEC HUS and occurs presumably due to the binding of Stx to its receptor on the glomerular basement membrane. Both glomerular injury and tubular injury in the form of acute tubular necrosis are common findings in STEC HUS [14, 15]. Cortical necrosis may occur in severe cases of STEC HUS. Neurologic symptoms including irritability, seizure and stroke are also reported in STEC HUS [16-18].

\section{Treatment}

Treatment for STEC HUS is largely supportive with the intent to normalize blood pressure, restore intravascular volume, recover electrolyte abnormalities, and maintain adequate nutrition. Gastrointestinal pain may be prominent and require either acetaminophen or even judicious use of morphine [19]. Patients may require transfusion of packed RBCs, however a clear threshold for transfusion has not been studied [6]. Platelet transfusions are not typical and are reserved for active bleeding or when preparing for an invasive procedure such as a catheter placement [13].

Key to the treatment of STEC HUS and prevention of renal complications is early fluid resuscitation and volume expansion. Current treatment using aggressive isotonic volume expansion is based on a protocol designed by Tarr et al. [19]. Crystalloid fluids should be administered in $20 \mathrm{ml} / \mathrm{kg}$ boluses until euvolemia is achieved and then subsequent fluid management is based on nutritional needs balanced against output [19].

Though case reports exist to demonstrate marginal effectiveness of plasma therapy in STEC HUS, there remains no clear role for plasma therapy in STEC HUS [20,21].

Renal replacement therapy may be required for some patients. Indications for dialysis parallel that of acute kidney injury of any cause: hyperkalemia, oligoanuria, uremia, metabolic acidosis, and/or volume overload. Both hemodialysis and peritoneal dialysis are suitable options [13]. Continuous renal replacement therapy may also be employed for more unstable patients with multiorgan involvement, severe fluid overload, or cardiovascular instability.
Antibiotic treatment for the prevention of typical HUS in children with STEC has not been shown to be effective. Specifically, literature has shown use of antibiotics such as fluoroquinolones and sulfa drugs to be more harmful than beneficial $[13,22,23]$.

Recently there has been interest in using anti-complement therapy (specifically eculizumab an anti-C5 antibody) in those patients with severe neurologic involvement [24]. The large outbreak of STEC HUS caused by E. coli O104:H4 that occurred in Germany in May 2011 tested this potential intervention. Eculizumab was used in a number of patients. The data are conflicting, however the bulk of the published data on HUS $[25,26]$ suggests that there are no short-term benefits of eculizumab in comparison to therapeutic plasma exchange in the treatment of STEC HUS. Therefore the role of eculizumab in this toxin-mediated disease remains unclear.

\section{Prognosis}

More than $80-90 \%$ of patients with STEC infection have a self-limited course, however those who develop typical HUS may require intensive care. Long-term sequelae may be present in up to $20 \%$ of children with typical HUS including chronic kidney disease, hypertension, diabetes, and neurological impairment $[16,26]$. Acute mortality from typical HUS is between 1 and 4\% $[1,16]$.

\section{Atypical HUS}

\section{Pathophysiology}

Atypical HUS (aHUS) is defined also as a microangiopathic anemia with thrombocytopenia and renal failure, however in this case there is most often (though not exclusively) no classic enteric prodrome [6, 27]. aHUS is caused by abnormalities in the alternate complement pathway (AP), resulting in uncontrolled complement activation and subsequent tissue damage.

The AP is constitutively active and functions as an arm of our innate immune system. Tight control of the AP is required to limit unregulated generation of both anaphylatoxins and the membrane attack complex [28]. Dysregulation of the alternate pathway of complement at the vascular endothelial cell surface results in the classic endothelial cell damage, platelet activation and thrombus formation that is seen in the other HUSs.

It has been shown that mutations in genes encoding proteins that regulate the AP or autoantibodies that inhibit AP complement regulatory proteins can be identified in approximately $60-70 \%$ of aHUS patients $[29,30]$. Both 
Table 2. Laboratory evaluation of a patient with suspected HUS - these laboratory studies may be modified based on the patient's clinical presentation

$\begin{array}{ll}\text { R/O STEC HUS } & \text { Stool or rectal swab: culture for STEC; PCR for Stx } \\ \text { serum: antibody testing } \\ \text { R/O Streptococcus pneumoniae infection } & \text { Blood cultures, pulmonary cultures } \\ \text { R/O thrombotic thrombocytopenia purpura } & \text { Plasma ADAMTS13 activity } \pm \text { inhibitor } \\ \text { R/O other secondary causes of TMA } & \text { Malignant hypertension, pancreatitis, malignancy, medication } \\ \text { R/O cobalamin metabolism abnormality } & \text { Homocysteine, methionine, urine organic acid testing } \\ \text { (i.e. methylmalonic aciduria) } & \text { Consider genetic testing for mutation in MMACHC gene } \\ \text { R/O rheumatologic disease } & \text { Antinuclear antibody, lupus anticoagulant, anti-phospholipid antibodies } \\ \text { R/O HIV } & \text { HIV serology } \\ \text { R/O pregnancy-associated TMA/HELLP syndrome } & \text { Pregnancy test, liver enzymes. } \\ \text { R/O complement pathway abnormality } & \text { C3, factor H, factor I, factor B serology } \\ & \text { Anti-factor H autoantibodies } \\ & \text { MCP - surface expression on leukocytes by FACS } \\ & \text { Gene mutation analysis for CFH, CFI, CFB, C3, CFHR5, MCP, evaluation } \\ & \text { for fusion proteins and CFHR1/3 deletion }\end{array}$

mutations that result in a quantitative deficiency of protein and mutations associated with a normal plasma level of functionally ineffective protein have been identified.

Complement factor $\mathrm{H}(\mathrm{CFH})$ mutations are the most common and account for $23-27 \%$ of identified mutations in registry patients in the USA and Europe [7, 8]. Membrane cofactor protein (MCP) mutations occur at a frequency of 5-7\%. Complement factor I (CFI) and complement component $\mathrm{C} 3(\mathrm{C} 3)$ occur at $4-8$ and $2-8 \%$ respectively. Gene mutations in complement factor $\mathrm{B}(\mathrm{CFB})$ and thrombomodulin (THBD) have also been noted (1-4 and $3-5 \%)[31,32]$.

The CFH-related proteins (CFHR) have also been associated with aHUS. In addition, non-allelic homologous recombination in the $\mathrm{CFH}$ and CFHR region results in hybrid proteins that are often poorly functioning and may affect the regulatory role of the native $\mathrm{CFH}$ protein. These mutations account for $1-3 \%$ of aHUS patients [30]. Finally, $6-10 \%$ of patients have an acquired risk for aHUS via anti-CFH [30, 33, 34].

$35-40 \%$ of individuals with a clinical scenario consistent with aHUS will have no demonstrable genetic mutation using current screening strategies. To further complicate this scenario, even when genetics are well described in a given family, the penetrance of disease is only $50 \%$ - with half of the family members with the same mutation remaining healthy [35].

aHUS is associated with a variety of disease triggers including organ transplantation, immunomodulatory agents and antiplatelet drugs [36-38]. Specifically an association between renal transplant, calcineurin inhibitor therapy, and humoral graft rejection [39-41] has been reported. Interestingly, $10-15 \%$ of aHUS cases appear to occur in conjunction with pregnancy or in the postpartum period [6].

\section{Clinical Manifestations and Diagnosis}

An estimated $10 \%$ of all HUS cases are classified as atypical or complement-mediated and are not associated with Stx $[6,42]$. Acute aHUS episodes are uncommonly associated with an enteric prodrome and classically manifest in severe hemolytic anemia, thrombocytopenia, and acute kidney injury [6]. In contrast to STEC HUS, aHUS may be precipitated by illness such as fever, upper respiratory tract infection, and non-bloody diarrhea. There have been reports of 0157:H7 E. coli triggering aHUS in pediatric patients with defined mutations [43]. Extrarenal, specifically central nervous system or visceral involvement, occurs in approximately $10-20 \%$ of patients [37, 43]. Recurrent, HUS should trigger the concern for aHUS [27]. The majority of patients will present their first episode of aHUS as a child $[44,45]$.

The diagnosis of aHUS requires that the clinician rule out potential confounding diagnoses (table 1). Most importantly, the patient must not meet criteria for STEC HUS and not meet criteria for thrombotic thrombocytopenia purpura. Current recommendations suggest that patients suspected of aHUS may require several laboratory screening studies (table 2) in order to rule out alternative HUS diagnoses. The goal is to arrive at an accurate diagnosis such that an appropriate therapy can be chosen. 
Fig. 1. An algorithm for the use of plasma therapy or the treatment of aHUS.

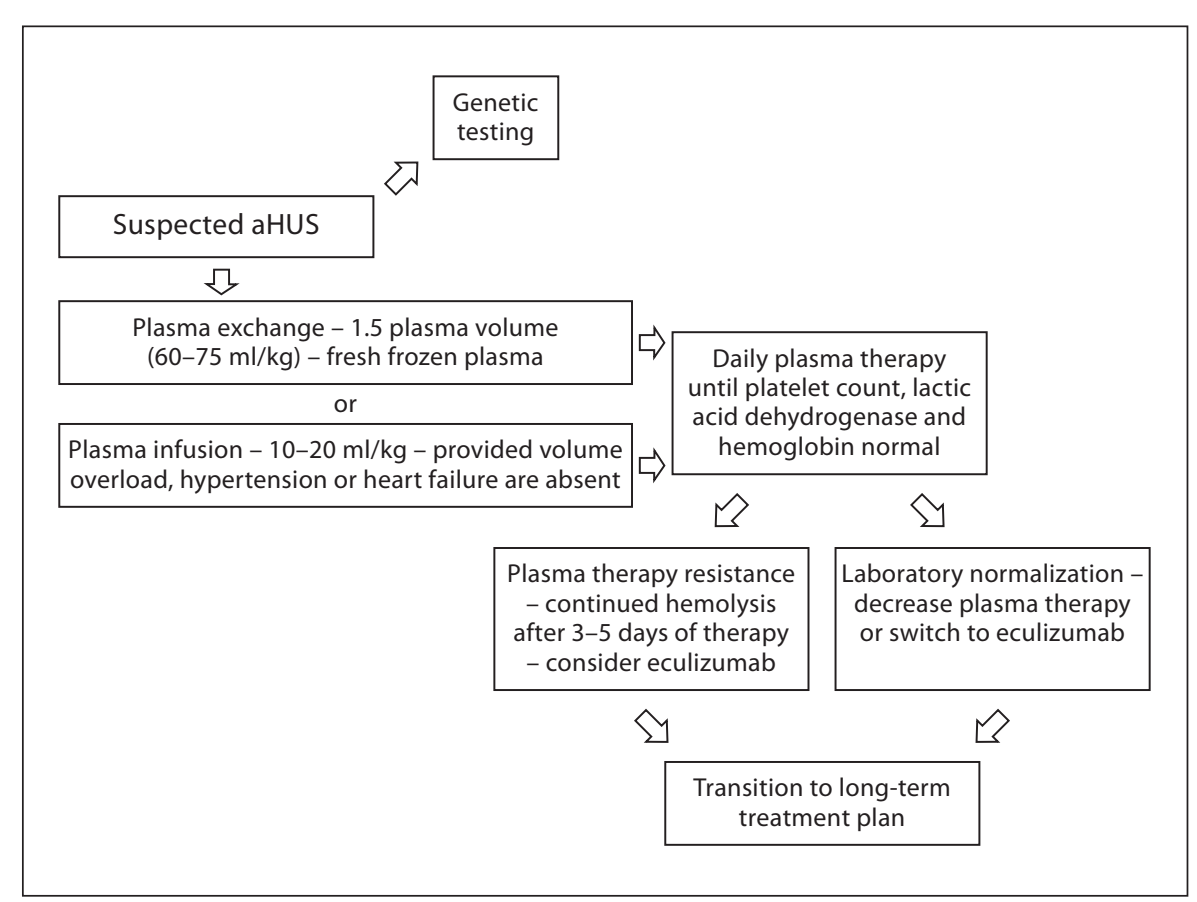

\section{Treatment}

As with STEC HUS, symptomatic management of volume, anemia and renal sequelae (e.g. normalization of blood pressure) should be a primary focus when aHUS is suspected. In conjunction with symptomatic care, plasma therapy (either exchange or infusion) has been the mainstay of acute therapy for aHUS. Although no randomized controlled trials have been completed to date, plasma exchange has been associated with at least a $25 \%$ decrease in mortality [46]. Plasma therapy should ideally be started within $24 \mathrm{~h}$ after establishing a presumed diagnosis of aHUS [20] Current recommendations suggest initiating exchange of 1.5 times plasma volumes with fresh frozen plasma or fresh frozen plasma mixed with albumin (approximately $65-70 \mathrm{ml} / \mathrm{kg}$ ) per session (fig. 1). Exchange should begin as a daily procedure for 5 days followed by 5 days per week for 2 weeks, and finally 3 times per week for 2 weeks [20]. Plasma therapy can then be reduced based on the patient's clinical response. It is unclear whether anticellular immunosuppressive agents must also be added to those patients that have anti-CFH antibodies in order to achieve remission [45]. Resolution of renal sequelae and hematologic normalization, resulting in either complete or partial remission, can occur in up to $60 \%$ of patients undergoing plasma exchange therapy $[44,45]$.
Trials using the anti-complement drug eculizumab have now been completed and the results reported in abstract form have been promising $[47,48]$. Published case studies and the adult and adolescent trial data abstracts suggests that eculizumab may be superior to plasma exchange for halting the hemolytic process [49]. Taken together, evidence suggests that eculizumab should be considered as an alternate initial treatment for aHUS. As with plasma therapy, if eculizumab is to be used for aHUS, it should be started as soon as possible to limit irreversible organ injury. Treatment doses are provided by the manufacturer. The duration of therapy continues to be debated.

When genetic mutation of one of the alternate pathway proteins produced in the liver is present, liver transplant may be an option. Liver transplantation has the potential to provide the physiologic means to correct the underlying genetic defect and prevent recurrence [50].

\section{Prognosis}

Until very recently, prognosis for aHUS has been poor. aHUS has been associated with death rates as high as $25 \%$ [42] and progression to ESRD in approximately $50 \%$ of patients $[6,51]$. Nearly $60-70 \%$ of all patients with mutations in $\mathrm{CFH}, \mathrm{CFI}$, or $\mathrm{C} 3$ mutations have relapsed or suffered end-stage renal disease within the first year of diagnosis, lost renal function during the presenting episode, 
or died during the presenting episode $[41,45]$. The introduction of anti-complement therapy for the treatment of aHUS is likely to alter the history of aHUS however to what extent remains to be seen.

When ESRD occurs and a renal transplant is undertaken, a high disease recurrence rate has been found (50\%) and the graft failure rate with recurrence has been $80-90 \%$. Protocols are now available to improve renal transplant outcome and they generally include both plasma therapy and eculizumab [52].

\section{Secondary HUS}

Lastly, there remains a subset of microangiopathic hemolytic anemias that do not fit into the above two classifications (table 1). These microangiopathies are known as the secondary HUSs and are typically named in association with the presumed precipitating disease (i.e. lupus-associated HUS or calcineurin inhibitor-associated HUS, etc.). This category includes all those diseases that cause HUS as a secondary phenomenon. Secondary HUSs have been described in association with a wide variety of diseases (most of which have either no or an unclear association with the AP) including non-STEC infectious agents (Streptococcus pneumonia and HIV), ma- lignancy, chemotherapy, bone marrow or solid organ transplantation, calcineurin inhibitors, pregnancy, malignant hypertension, systemic lupus erythematous and other rheumatologic diseases and disorders of cobalamin metabolism [6, 37, 53-55]. In general, the treatment for a secondary HUS is to treat the primary disorder and support the patient with fluid, blood or antihypertensive therapy as necessary. There are no data to support a more specific treatment of this type of microangiopathy at this time and it remains unclear whether anti-complement therapy may be useful in this setting.

In conclusion, the thrombotic microangiopathies are a heterogenous group of diseases with similar presenting characteristics that have both different etiologic mechanisms and different ideal treatment regimens. Despite the absence of specific markers to distinguish these diseases and the difficulty in separating them clinically, it is nonetheless supremely important to attempt to categorize the HUSs according to one of these subsets in order to deliver optimal care.

\section{Disclosure Statement}

Dr. Nester is on Alexion Pharmaceutical's International Advisory Board for Atypical Hemolytic Uremic Syndrome.

\section{References}

1 Tarr PI, Gordon CA, Chandler WL: Shigatoxin-producing Escherichia coli and haemolytic uraemic syndrome. Lancet 2005; 365:1073-1086

-2 Karpman D, Sartz L, Johnson S: Pathophysiology of typical hemolytic uremic syndrome. Semin Thromb Hemost 2010;36:575-585.

3 Karmali MA, Steele BT, Petric M, Lim C: Sporadic cases of haemolytic-uraemic syndrome associated with faecal cytotoxin and cytotoxin-producing Escherichia coli in stools. Lancet 1983;1:619-620.

4 Karmali MA: Infection by Shiga toxin-producing Escherichia coli: an overview. Mol Biotechnol 2004;26:117-122.

5 Karmali MA: Host and pathogen determinants of verocytotoxin-producing Escherichia coli-associated hemolytic uremic syndrome. Kidney Int Suppl 2009:S4-S7.

6 6 Noris M, Remuzzi G: Hemolytic uremic syndrome. J Am Soc Nephrol 2005;16:10351050.

7 Lynn RM, O’Brien SJ, Taylor CM, et al: Childhood hemolytic uremic syndrome, United Kingdom and Ireland. Emerg Infect Dis 2005;11:590-596.
McKee ML, O'Brien AD: Investigation of en- 13 Bitzan M, Schaefer F, Reymond D: Treatterohemorrhagic Escherichia coli O157:H7 adherence characteristics and invasion potential reveals a new attachment pattern shared by intestinal E. coli. Infect Immun 1995;63:2070-2074.

$\checkmark 9$ Kaper JB, Nataro JP, Mobley HL: Pathogenic Escherichia coli. Nat Rev Microbiol 2004;2: 123-140.

10 Zoja C, Corna D, Farina C, et al: Verotoxin glycolipid receptors determine the localization of microangiopathic process in rabbits given verotoxin-1. J Lab Clin Med 1992;120: 229-238.

11 Nestoridi E, Tsukurov O, Kushak RI, et al: Shiga toxin enhances functional tissue factor on human glomerular endothelial cells: implications for the pathophysiology of hemolytic uremic syndrome. J Thromb Haemost 2005;3:752-762.

12 Riley LW, Remis RS, Helgerson SD, et al: Hemorrhagic colitis associated with a rare Escherichia coli serotype. N Engl J Med 1983; 308:681-685. ment of typical (enteropathic) hemolytic uremic syndrome. Semin Thromb Hemost 2010;36:594-610.

14 Kaneko K, Kiyokawa N, Ohtomo Y, et al: Apoptosis of renal tubular cells in Shiga-toxin-mediated hemolytic uremic syndrome. Nephron 2001;87:182-185.

15 Van Setten PA, van Hinsbergh VW, van der Velden TJ, et al: Effects of TNF- $\alpha$ on verocytotoxin cytotoxicity in purified human glomerular microvascular endothelial cells. Kidney Int 1997;51:1245-1256.

16 Oakes RS, Siegler RL, McReynolds MA, et al: Predictors of fatality in postdiarrheal hemolytic uremic syndrome. Pediatrics 2006;117: 1656-1662.

17 Taylor CM, White RH, Winterborn MH, Rowe B: Haemolytic-uraemic syndrome: clinical experience of an outbreak in the West Midlands. Br Med J (Clin Res Ed) 1986; 292:1513-1516.

18 Bale JF Jr, Brasher C, Siegler RL: CNS manifestations of the hemolytic-uremic syndrome. Relationship to metabolic alterations and prognosis. Am J Dis Child 1980;134:869-872. 
$\checkmark 19$ Ake JA, Jelacic S, Ciol MA, et al: Relative nephroprotection during Escherichia coli O157:H7 infections: association with intravenous volume expansion. Pediatrics 2005; 115:e673-e680.

20 Ariceta G, Besbas N, Johnson S, et al: Guideline for the investigation and initial therapy of diarrhea-negative hemolytic uremic syndrome. Pediatr Nephrol 2009;24:687-696.

21 Kavanagh D, Richards A, Atkinson J: Complement regulatory genes and hemolytic uremic syndromes. Annu Rev Med 2008;59: 293-309.

22 Michael M, Elliott E, Craig J, et al: Interventions for hemolytic uremic syndrome and thrombotic thrombocytopenic purpura: a systematic review of randomized controlled trials. Am J Kidney Dis 2009;53:259-272.

23 Wong CS, Jelacic S, Habeeb RL, et al: The risk of the hemolytic-uremic syndrome after antibiotic treatment of Escherichia coli O157:H7 infections. N Engl J Med 2000;342:19301936.

-24 Lapeyraque AL, Malina M, Fremeaux-Bacchi V, et al: Eculizumab in severe Shiga-toxin-associated HUS. N Engl J Med 2011;364: 2561-2563.

25 Kielstein JT, Beutel G, Fleig S, et al: Best supportive care and therapeutic plasma exchange with or without eculizumab in Shigatoxin-producing E. coli O104:H4 induced haemolytic-uraemic syndrome: an analysis of the German STEC-HUS registry. Nephrol Dial Transplant 2012;27:3807-3815.

-26 Siegler R, Oakes R: Hemolytic uremic syndrome; pathogenesis, treatment, and outcome. Curr Opin Pediatr 2005;17:200-204.

27 Noris M, Remuzzi G: Atypical hemolytic uremic syndrome. N Eng J Med 2009;361: 1676-1687.

28 Mackay IR, Rosen FS, Walport MJ: Complement. N Engl J Med 2001;344:1058-1066.

29 Maga TK, Nishimura CJ, Weaver AE, et al: Mutations in alternative pathway complement proteins in American patients with atypical hemolytic uremic syndrome. Hum Mutat 2010;31:E1445- E1460.

- 30 Noris M, Caprioli J, Bresin E, et al: Relative role of genetic complement abnormalities in sporadic and familial aHUS and their impact on clinical phenotype. Clin J Am Soc Nephrol 2010;5:1844-1859.

-31 Goicoechea de Jorge E, Harris CL, EsparzaGordillo J, et al: Gain-of-function mutations in complement factor B are associated with atypical hemolytic uremic syndrome. Proc Natl Acad Sci USA 2007;104:240-245.
32 Delvaeye $\mathrm{M}$, Noris $\mathrm{M}$, De Vriese A, et al: Thrombomodulin mutations in atypical hemolytic-uremic syndrome. N Engl J Medl 2009;361:345-357.

33 Moore I, Strain L, Pappworth I, et al: Association of factor $\mathrm{H}$ autoantibodies with deletions of CFHR1, CFHR3, CFHR4, and with mutations in $\mathrm{CFH}, \mathrm{CFI}, \mathrm{CD} 46$, and $\mathrm{C} 3$ in patients with atypical hemolytic uremic syndrome. Blood 2010;115:379-387.

34 Lee BH, Kwak SH, Shin JI, et al: Atypical hemolytic uremic syndrome associated with complement factor $\mathrm{H}$ autoantibodies and CFHR1/CFHR3 deficiency. Pediatr Res 2009;66:336-340.

35 Sullivan M, Erlic Z, Hoffmann MM, et al: Epidemiological approach to identifying genetic predispositions for atypical hemolytic uremic syndrome. Ann Hum Genet 2010;74: $17-26$.

36 Ruggenenti P, Noris M, Remuzzi G: Thrombotic microangiopathy, hemolytic uremic syndrome, and thrombotic thrombocytopenic purpura. Kidney Int 2001;60:831-846.

37 Besbas N, Karpman D, Landau D, et al: A classification of hemolytic uremic syndrome and thrombotic thrombocytopenic purpura and related disorders. Kidney Int 2006;70: 423-431.

38 Zakarija A, Bennett C: Drug-induced thrombotic microangiopathy. Semin Thromb Hemost 2005;31:681-690.

39 Ruggenenti P: Post-transplant hemolyticuremic syndrome. Kidney Int 2002;62:10931104.

40 Zarifian A, Meleg-Smith S, O’Donovan R, et al: Cyclosporine-associated thrombotic microangiopathy in renal allografts. Kidney Int 1999;55:2457-2466.

-41 Karthikeyan V, Parasuraman R, Shah V, et al: Outcome of plasma exchange therapy in thrombotic microangiopathy after renal transplantation. Am J Transplant 2003;3: 1289-1294.

42 Kaplan BS, Meyers KE, Schulman SL: The pathogenesis and treatment of hemolytic uremic syndrome. J Am Soc Nephrol 1998;9: $1126-1133$

43 Sellier-Leclerc AL, Fremeaux-Bacchi V, Dragon-Durey MA, et al: Differential impact of complement mutations on clinical characteristics in atypical hemolytic uremic syndrome. J Am Soc Nephrol 2007;18:23922400
44 Caprioli J, Noris M, Brioschi S, et al: Genetics of HUS: the impact of MCP, CFH, and IF mutations on clinical presentation, response to treatment, and outcome. Blood 2006;108: 1267-1279.

45 Loirat C, Noris M, Fremeaux-Bacchi V: Complement and the atypical hemolytic uremic syndrome in children. Pediatr Nephrol 2008;23:1957-1972.

46 George JN: How I treat patients with thrombotic thrombocytopenic purpura: 2010 Blood 2010;116:4060-4069.

47 Legendre C, Furman RR, Sheerin NS, et al: Safety and efficacy of eculizumab in aHUS resistant to plasma therapy: interim analysis from a phase II trial. FC 406. 43rd Annual Meeting of the American Society of Nephrology, Denver, Colo, November 16-21, 2010

48 Muus P, Douglas K, Hourmant M, et al: Safety and efficacy of eculizumab in aHUS patients on chronic plasma therapy: Interim analysis of a phase II trial. Poster 1274. 43rd Annual Meeting of the American Society of Nephrology, Denver, Colo, November 16-21, 2010

49 Vilalta R, Lara E, Madrid A, et al: Long-term eculizumab improves clinical outcomes in atypical hemolytic uremic syndrome. Pediatr Nephrol 2012;27:2323-2326.

-50 Remuzzi G, Ruggenenti P, Colledan M, et al: Hemolytic uremic syndrome: a fatal outcome after kidney and liver transplantation performed to correct factor $\mathrm{h}$ gene mutation. Am J Transplant 2005;5:1146-1150.

51 Constantinescu AR, Bitzan M, Weiss LS, et al: Non-enteropathic hemolytic uremic syndrome: causes and short-term course. Am J Kidney Dis 2004;43:976-982.

52 Nester C, Stewart Z, Myers D, et al: Pre-emptive eculizumab and plasmapheresis for renal transplant in atypical hemolytic uremic syndrome. Clin J Am Soc Nephrol 2011;6: 1488-1494.

53 Sharma AP, Greenberg CR, Prasad AN, Prasad C: Hemolytic uremic syndrome secondary to cobalamin C disorder. Pediatr Nephrol 2007;22:2097-2103.

54 Izzedine $\mathrm{H}$, Rixe O, Billemont B, et al: Angiogenesis inhibitor therapies: focus on kidney toxicity and hypertension. Am J Kidney Dis 2007;50:203-218.

55 Bollee G, Patey N, Cazajous G, et al: Thrombotic microangiopathy secondary to VEGF pathway inhibition by sunitinib. Nephrol Dial Transplant 2009;24:682-685. 